\title{
Kinetics of Gold Nanorods Formation in Solution and Application on Aspect Ratio Tuning
}

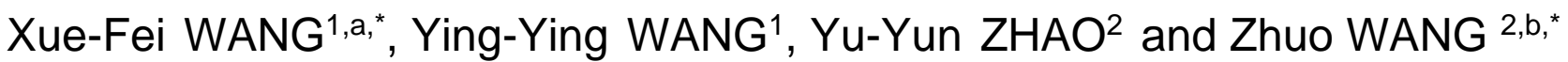

${ }^{1}$ School of Chemistry and Chemical Engineering University of Chinese Academy of Sciences Beijing 100049, China

${ }^{2}$ College of Science, Beijing University of Chemical Technology Beijing 100029 China

ae-mail: wangxf@ucas.ac.cn, be-mail:wangzhuo77@mail.buct.edu.cn

\section{Keywords: Gold nanorod, Aspect ratio, Morphology tuning, Growth mechanism}

\begin{abstract}
For the gold nanorods synthesis by seed-mediated method, the overgrowth goes against the shape controlling for the final product. Here, through optimizing the growth solution condition, the nanorods shape could be tuned effectively by just adjusting the seed amount introduced initially. Further, the growth-stop experiment reveals that the final nanorods with different shapes have a similar size parameter at their respective turning points (TP) of the overgrowth process, indicating that all nanorods in solution grow following a common morphology evolution trajectory but terminate at different points, which have certain aspect ratio. Additionally, the morphology evolution of gold nanorods could be simulated quantitatively using the classic chemical dynamic theory, which also opens a pathway to tune the aspect ratio of product accurately.
\end{abstract}

\section{Introduction}

Gold nanorods, with a variable surface plasma resonance band at visiable/NIR, have attracted much attention in many fields, such as thermal therapy[1-3], cellar imaging[4,5], optical polarizer[6], drug delivery[7,8]. Advanced to other anisotropic metal nanoparticles, gold nanorods can be synthesized in mass production just by simple chemical reaction under ambient condition, and the surface plasma resonance band is variable[9]. Even though, lots of efforts, for example, adjusting the reactants concentrations[9,10], alter surfactant[11], reshaping by etching[12] or heating[12,13], have been made to optimize the synthesis protocol because of the uncertain morphology of the final products. Actually, for the seed mediated synthesis in solution gold nanorods perform a complicate mechanism. Previous literatures and our studies revealed that the formation of gold nanorods consists of at least two stages: seeds develop into nanorods with fast aspect ratio (AR) increase and subsequently the formed nanorods grow bigger with AR decreasing[14,15]. Based on this mechanism, we have developed the growth-stop technique to tune the AR of gold nanorods[14]. In brief, by adding chemical inhibitor to deplete the residual gold salt in solution, the growth process was inhibited totally at certain stage and nanorods with desirable shape were obtained. The technique gave an effect controlling on the aspect ratio of final product, however, it leads to a low yield as for the depletion of remainder gold salt.

It is clear that the overgrowth at the second stage should be ascribed to the deposition of remainder gold salt in solution. Thus, if introducing additional seeds to consume the remainder gold atoms, instead of forming clusters under depletion, not only the overgrowth will be inhibited but also the gold salt is utilized fully. And also, the additional seed means more products in solution. Inspired by this idea, an approach to control the AR of gold nanorods by adjusting the seed amount was developed. Additionally, the serial size parameters of the particles also provide an opportunity to insight into the growth mechanism of gold nanorods, which is not clear till now. 


\section{Experimental}

\section{Materials}

Chloroauric acid $\left(\mathrm{HAuCl}_{4}\right)$, ascorbic acid (AA), sodium borohydride $\left(\mathrm{NaBH}_{4}\right)$, and silver nitrate $\left(\mathrm{AgNO}_{3}\right)$ were purchased from Aldrich. Cetyltrimethylammonium bromide (CTAB) was purchased from Alfar. All reagents were used as received. Deionized water (>18.0 M $\Omega$ ) from a Millipore water purification system was used for all experiments.

\section{Gold Nanorods Synthesis.}

For seed synthesis, $0.15 \mathrm{~mL}$ of icecold $10 \mathrm{mM} \mathrm{NaBH}_{4}$ was injected into $2 \mathrm{~mL}$ of rapidly stirred solution of $0.25 \mathrm{mM} \mathrm{HAuCl}_{4}$ in $0.1 \mathrm{M} \mathrm{CTAB}$. The pale brown solution left for at least $2 \mathrm{~h}$ before use to make sure all $\mathrm{NaBH}_{4}$ dissociated. A typical NRs synthesis involves a $2.0 \mathrm{~mL}$ aqueous solution consisting of $0.1 \mathrm{M} \mathrm{CTAB}$ prepared in a $1 \mathrm{~cm}$ quartz optical cell. Also, $50 \mu \mathrm{L}$ of $20 \mathrm{mM}$ $\mathrm{HAuCl}_{4}$ solution, $20 \mu \mathrm{L}$ of $10 \mathrm{mM} \mathrm{AgNO}$, and $28 \mu \mathrm{L} 50 \mathrm{mM} \mathrm{AA}$ were added to the cell in turn followed by gentle mixing. AA amount is determined by the $[\mathrm{AA}]:\left[\mathrm{HAuCl}_{4}\right]=3: 2$ ratio. Seed solution was injected into the mixture under stirring to initiate the growth of gold nanorods. For the aspect ratio controlling, different volumes of seed solution, 7, 20, 30 and $50 \mu \mathrm{L}$, was added.

\section{Growth-Stop Performance}

To get the morphology information of the particle at the maximal plasma resonance wavelength, growth-stop technique was used: Monitoring the growth solution by repeating spectral measurement, and at the desirewavelength $0.1 \mathrm{~mL}$ fresh prepared ice-cold $\mathrm{NaBH}_{4}(10 \mathrm{mM})$ was injected into the growth solution under vigorous stirring. More details of the operation have been provided in our previous work[14].

\section{Measurement}

Absorption spectra in the range of 450-900 nm were recorded with a Hitachi $3310 \mathrm{UV}-\mathrm{Vis}$ spectrophotometer at room temperature using quartz cell of $10 \mathrm{~mm}$ path length. An automatically repetitive measurement has been carried out to record the spectral variation over time, and one spectrum was recorded per-minute with a scan speed of $1200 \mathrm{~nm} / \mathrm{min}$. TEM images were acquired with a JEOL JEM-1011 transmission electron microscope. The samples for TEM were centrifuged $(10000 \mathrm{rpm} \times 10 \mathrm{~min})$ twice to remove the surfactant, and redispersed into water.

\section{Results and Discussion}

In present work, the synthesis of gold nanorods follows the traditional seed-mediated protocol [9] with modification: $0.5 \mathrm{mM} \mathrm{HAuCl} 4,0.75 \mathrm{mM}$ ascorbic acid and $0.1 \mathrm{mM}$ silver nitrate in $0.1 \mathrm{M}$ CTAB (See Experimental section for details). Noteworthily, the ratio of $\mathrm{HAuCl}_{4}$ to ascorbic acid of 1:1.5 makes sure that gold salt can be reduced into atoms totally. Fig. 1 gives a series of representative absorption spectra of the final growth solution, where varying seeds solution addition, $7,20,30$, and $50 \mu \mathrm{L}$ results in various longitudinal plasmon resonance peaks located around 650 , 700,750 and $800 \mathrm{~nm}$, respectively, exhibiting strong dependence of the longitudinal plasmon resonance (LPR) on the seed amount applied. Furthermore, from the corresponding TEM images (Fig.2) the well-defined rod-shaped particles are observed, and their averaged length and width can be achieved through the statistical analysis of the images, giving the values of $45.1 \times 20.4 \mathrm{~nm}$, $38.8 \times 13.6 \mathrm{~nm}, 36.8 \times 11.7 \mathrm{~nm}$, and $32.0 \times 9.0 \mathrm{~nm}$. This result demonstrates the feasibility of the aspect ratio tuning via seed amount. Juncheng and coworkers found that longer aging time of the seed solution results in gold nanorods with lower aspect ratio and shorter plasma resonance wavelength [16]. This is in consistent with our results, as for the long aging process does result in aggregation of the synthesized seed, which leads to decreasing of the seed number in solution. 


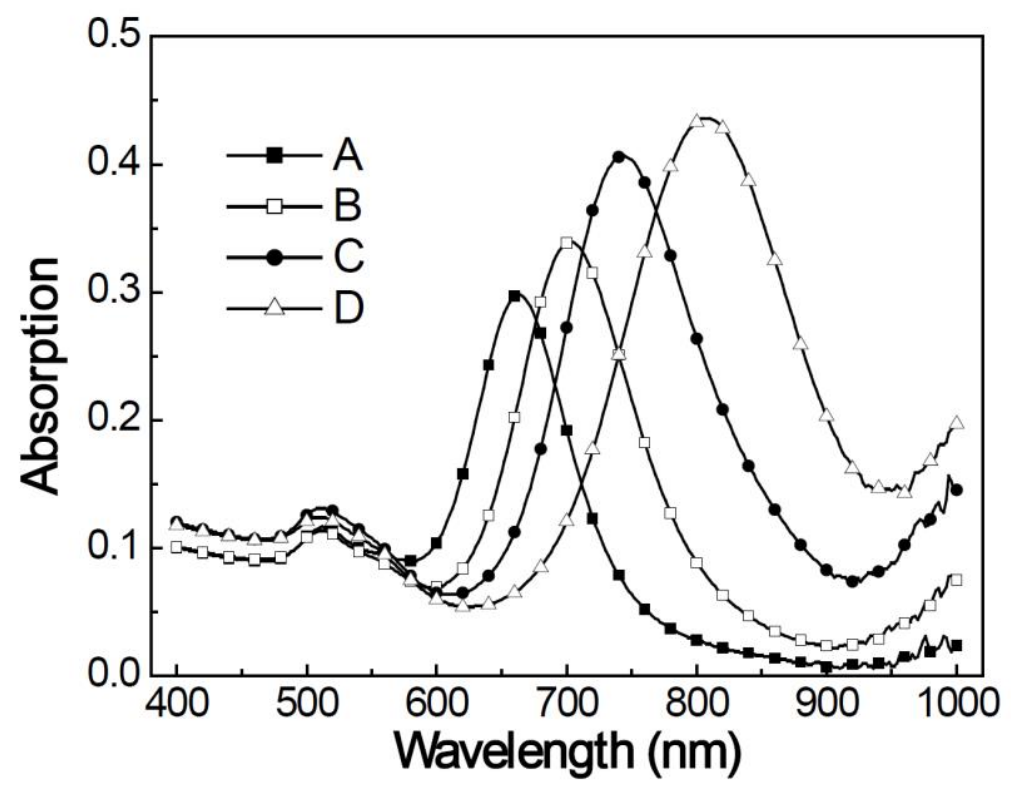

Fig. 1. Absorption spectra of the final collected gold nanorods. A, B, C and D ref to the samples with varying seed solution volumes, $7,20,30$ and $50 \mu \mathrm{L}$, respectively.

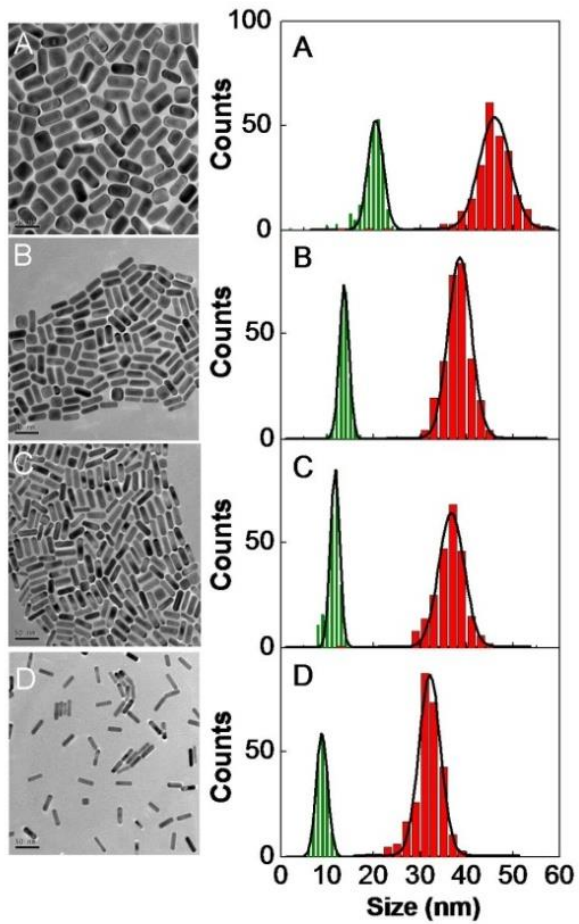

Fig. 2. (Left)TEM images of samples in Fig. 1. (Right) Histograms of width (green) and length (red). In addition, a Gaussian fit has been performed to obtain the center of the distribution. Scale bars represent $50 \mathrm{~nm}$.

Despite the discrepancy of the particle size, the yield of Au atom should be the same in theory. Suppose every seed in the solution hasa an opportunity to become a nanorod and all gold precursors in solution have been deposited on nanorods, the following relationship could be given:

$$
\mathrm{V}_{\text {rod }} \times \rho_{\text {rod }} \times \mathrm{V}_{\text {seed }} \times \mathrm{c}_{\text {seed }}=\mathrm{M}_{\text {Au }} \quad \text { or } \quad \mathrm{V}_{\text {rod }} \times \mathrm{V}_{\text {seed }} \propto \mathrm{M}_{\text {Au }}
$$

Where $\mathrm{V}_{\text {rod }}$ is the average volume of nanorod, $\rho_{\text {rod }}$ is the density, $\mathrm{V}_{\text {seed }}$ is the volume of seed solution added (proportional to seed number), $\mathrm{c}_{\text {seed }}$ is the seed concentration, and $\mathrm{M}_{\mathrm{Au}}$ is the gross mass of gold precursor (the same for four cases). According to the parameters of size (Fig.2) and 
seed solution volume above, the ratios of $\mathrm{V}_{\text {rod }} \times \mathrm{V}_{\text {seed }}$ for the four samples are $\mathrm{A}: \mathrm{B}: \mathrm{C}: \mathrm{D}=1.10: 1.07: 1.13: 0.97$, demonstrating the same yield $(\sim 1)$ in four cases. Unfortunately, we failed in elucidating the quantitative relationship between the seed solution volume and particle size here, because the seed concentration is unknown.

To exploring the growth mechanism, the real-time spectral monitoring has been used to follow the growth process. In agreement to previous report[14,17], the spectra perform a fast red shift firstly and then a gradual blue shift, reaching final products with different LPR bands. Obviously, the blue shifts are corresponded to the AR decreasing, which should be attributed to the overgrowth[14,18], i. e. a process that the rods become fat from slender ones. Interestingly, a same turning wavelength at $840 \mathrm{~nm}$ was observed in four cases. Further, to get the morphology of nanorods at the turning point, the growth-stop technique[14] was used timely at the maximal LPR wavelength to inhibit the deposition. Fig.4 shows the spectral evolutions before growth-stop and corresponding TEM images of the collected particles. Here, for four cases A, B, C, and D, the identical initial conditions corresponding to those in Fig. 1 and Fig. 2 have been applied. Further, the size statistics based on the TEM images (Fig.3) give the size parameters at length $\times$ width: $27.0 \times 7.9 \mathrm{~nm}, 26.4 \times 8.4 \mathrm{~nm}, 27.4 \times 8.2 \mathrm{~nm}$ and $27.6 \times 7.8 \mathrm{~nm}$ for A, B, C, and D, respectively. This closed values suggest that in four systems the gold nanorods passes through a common point, at which the particles have the same morphology. That is to say, the serial final products of different sizes in Fig. 2 are the results of growth from ones with the same morphology. Further, taking account into the whole spectral evolutions(Fig.3), before the TP particles perform growth from seeds into slender rods (red-shift LPR), then after that the as-formed nanorods become bigger and fatter (blue-shift LPR). On the other hand, despite of the seed concentration varying in four cases, for each seed in solution, it has the totally identical initial micro-environment, i.e. the same solvent surrounding and the same reactants concentration. Having the same initial environment and then passing through a common critical point, it is logically predicated that all gold nanorods grow along a common growth trajectory, which figures out the morphology evolution of individual nanorods. Easily understanding, the variation of the final morphology is because of the exhaustion of gold precursor in solution, which defines the termination position of growth on the trajectory. For easy composition, all size data are summarized in table 1 .

Table 1. Summary of the gold nanorod size parameters in Fig.2 (final nanorods) and Fig.4 (turning point). All data are in unit of $\mathrm{nm}$.

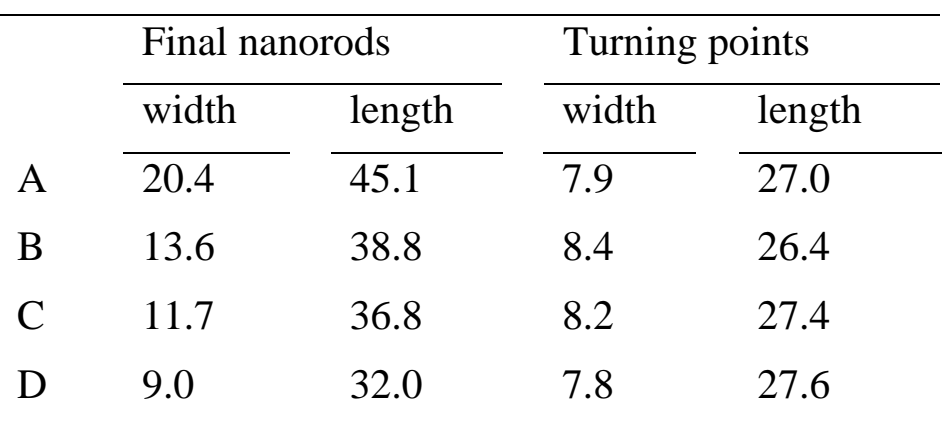



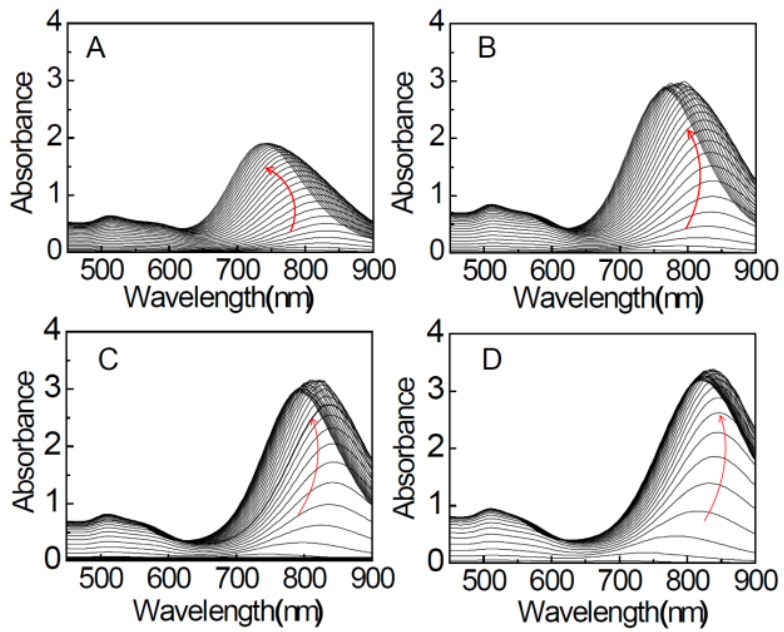

Fig.3. The first thirty absorption spectra of the growth solutions with one minute interval. The arrows indicate the spectral evolution.
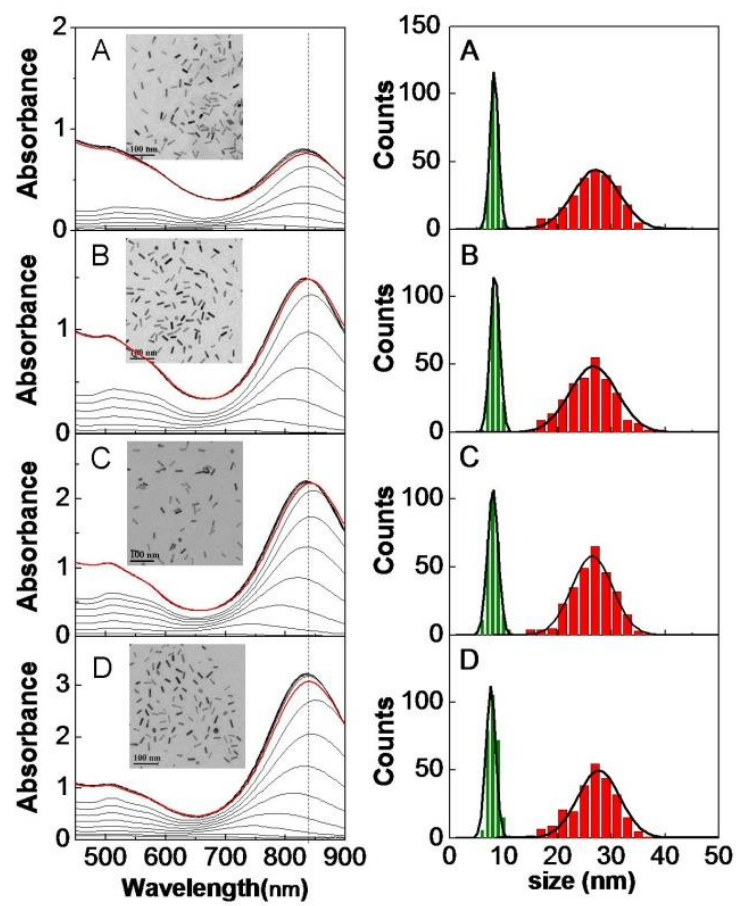

Fig.4. (Left)Spectral evolution of growth-stop at the maximal wavelength $(840 \mathrm{~nm}$, singned by dashed line). The curves in red line suggest the point of $\mathrm{NaBH}_{4}$ addition. $\mathrm{A}, \mathrm{B}, \mathrm{C}$ and $\mathrm{D}$ have the totally identical initial condition with the correspond cases in Fig. 1. Insets are the TEM images of the collected nano-particles. Scale bars represent $100 \mathrm{~nm}$. (Righ) Histograms of width (green) and length (red) of gold nanorods. Corresponding Gaussian fits are plotted.

It is known that the single crystal gold nanorod has octagonal column morphology, with alternated $\{100\}$ and $\{110\}$ lateral facets[19], but Enrique and coworkers observed a higher-index lateral facets $\{250\}$, and predicted a plausible transition from the $\{250\}$ to $\{110\}[20]$. This is very probably relevant to the two-stage growth model above, in which nanorods before and after the TP have different type of lateral facets, $\{250\}$ and $\{110\}$, respectively. It is clear that the rod-shape formation is due to the difference of deposition rate on lateral and end facets[21], i.e. the fast deposition rate on the $\{250\}$ lateral facets and relatively slower one on the $\{111\}$ end facets. The consequent $A R$ decreasing should be also ascribed to the relative slow rate on the $\{110\}$ facet than 
$\{250\}$. Kinetically, the facet transition from $\{250\}$ to $\{110\}$ should be a successive and gradual process, which thus leads to a spectral evolution above $[22,23]$.

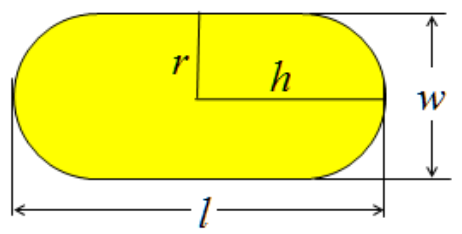

Scheme 1. Size parameters of the gold nanorod.

Actually, the growth process is a redox reaction of gold salt and ascorbic acid on the surface of nanoparticle[24], with product of gold atoms, which result in the size increasing of the nanorods. Therefore, the growth dynamics can be modeled by calculating the deposition rate along the longitudinal and transverse axils respectively according to chemical reaction dynamics functions[25]:

$$
\begin{aligned}
& d r=d w / 2=k_{r}\left[A u^{3+}\right][A A] d t \\
& d h=d l / 2=k_{h}\left[A u^{3+}\right][A A] d t
\end{aligned}
$$

Where, as shown in scheme $1, w, l, h$ and $r$ are the width, length, half length and radius of nanorods, respectively, as well as $l=2 h$ and $w=2 r k r$ and $k h$ (with unit $n m \cdot M^{-1} \cdot s^{-1}$ ) are the growth rate constant of $r$ and $h$, respectively $\left[\mathrm{Au}^{3+}\right]$ is the concentration of gold precursor in form of $\mathrm{AuBr}_{2}{ }^{1-}[25,26]$, and $[\mathrm{AA}]$ is the ascorbic acid concentration.

According to the Arrhenius' equation which describes the basic chemical kinetics[27], $\mathrm{k}_{\mathrm{r}}$ and $\mathrm{kh}_{\mathrm{h}}$ should be an exponential function of the activation energy $\Delta \mathrm{G}$. As known, the growth process can be looked as a self-catalystic reaction, in which the reaction of $\mathrm{AuBr}_{2}{ }^{1-}$ and $\mathrm{AA}$ is under catalysis of the surface of gold nanorods. This process is complicated, however, it should be a relative to the surface form crystal surface activity energy $\left(\Delta \mathrm{G}_{\mathrm{sae}}\right)$,

$$
\left.k_{r}=A \exp \measuredangle G_{\text {sae }} / R T\right)
$$

As discussed above, with the particle growth and facet transition from $\{250\}$ to $\{110\}$, its surface activity energy is charging gradually. Here, suppose latticactivity energy takes linear decreaseupon radius increase,

$$
\Delta G_{s a e}=\Delta G_{s a e}^{0}-k_{r}^{\prime}\left(r-r_{0}\right) R T=\Delta G_{s e}^{0}-k_{r}\left(r-w_{0}\right) 2
$$

Where, $k_{r}{ }^{\prime}$ is the decrease rate of $\Delta G_{s a e}$ upon $r$ increasing, with unit of $\mathrm{J} \mathrm{mol}^{-1} . n m^{-1}$, and $r o$, wo the initial value of radius and width of nanoparticles, $\Delta G_{s a e}{ }^{0}$, surface energy when $\mathrm{r}=\mathrm{r}_{0}$. Therefore, equation 1 can be written as,

$$
d w / 2=k_{r 0} \exp \left[k_{r}^{\prime}\left(w-w_{0}\right) / 2 R T\right][A u][A A] d t
$$

Where $k_{r}=k_{r} 0$ when $r=r_{0}$, i.e. $w=w o$. In addition, due to the invariable end facet $\{111\}$, the end activity energy is constant, and $k_{h}$ is also constant $k_{h}=k_{h 0}$. According to Equ. 2 and Equ.3, the relationship between length and width can be achieved

$$
l=l_{0}+\frac{2}{k_{r}^{\prime}} \frac{k_{h 0}}{k_{r 0}}\left\{1-\exp \left[-k_{r}^{\prime}\left(w-w_{0}\right) / 2 R T\right]\right\}
$$

Where $l_{0}$ and $w_{0}$ are the initial size parameters of particle, i.e the seed diameter, $l_{0}=w_{0}=3$ $\mathrm{nm}[28,29]$. Actually, the small values of $l_{0}$ and wo really bring about little effect on the final particle size estimation. Reaction temperature $\mathrm{T}=300 \mathrm{~K}$.

Eq. 4 gives a relationship between the length and width during growth process. As discussed previously, each particle in solution has the same growth trajectory, in spite of the different terminal point due to variation of average amount of gold atoms on each seed. Therefore, the size parameters of the synthesized nanorods here should all obey the relationship of equation 4. To test this hypothesis, the parameters of length and width (Table 1) corresponding to true particles, including those presented in Fig. 2 and the average value in Fig.4, are plotted in Fig.5, and are fitted using equation 4 . The good fit to the measured data by the estimated equation indicates the rationality of 
our prediction on the growth mechanism. Here, the higher ratio of the initial rate $\left(k_{h o} / k_{r 0}=7.1\right)$ shows that for seeds the growth rate at the both ends is much faster than that of the later facet, thus resulting in the formation of slender rods from seed $\left(l_{0}=w_{0}=3 \mathrm{~nm}\right)$.However, the exponential increasing of $k_{r}$ results in the rate ratio decreasing $\left(k_{h} / k_{r}<7.1\right)$ and correspondingly aspect ratio decreasing with a maximal $A R=3.4$. Since the average atom number on individual nanorod is statistically negative proportional to seed amount, the seed amount may determine the position on the trajectory, where the precursor exhausts, the growth terminates and the particles have certain AR. In addition, the aspect ratios driven from the fitting data are shown in Fig.5, and a turning point as a function of width can be observed clearly, with highest $\mathrm{AR}=3.4$, in agreement with previous report[28]. According to the simulation, the corresponding aspect ratio data as function of the width can be generated, as shown in Fig. 5. Here aspect ratio shows range from the maximal value 3.4 to 2 or smaller, that is to say, we can synthesize gold nanorods with any aspect ratio within this region just by adjusting the seed amount. Further studies to elucidate the quantum relation between seed amount and finial particle morphology are in progress.

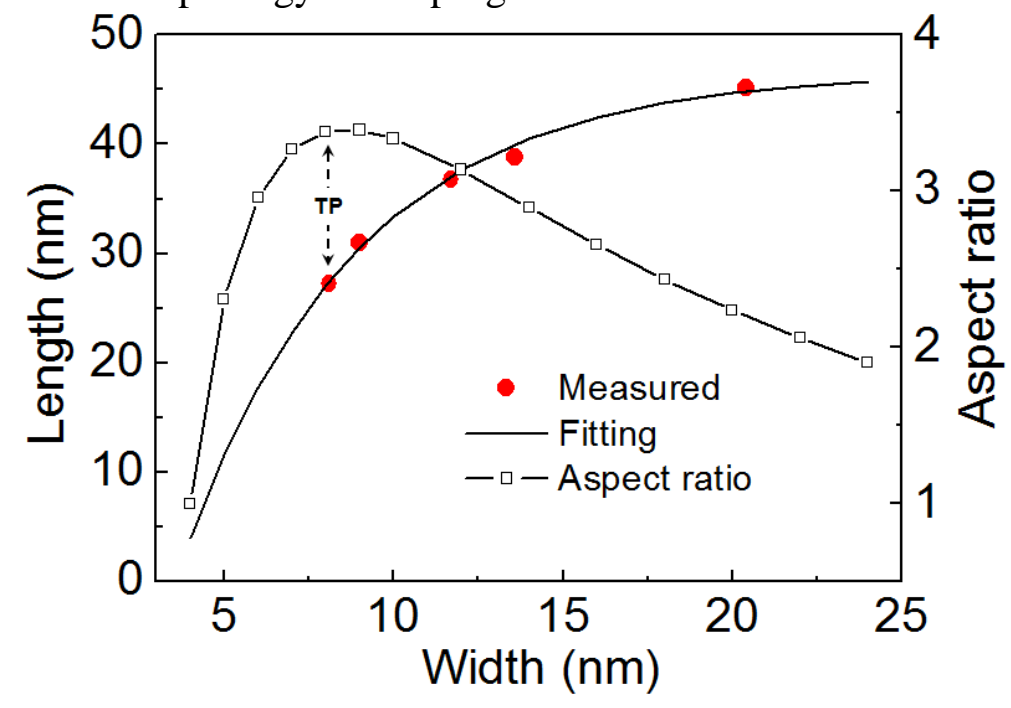

Fig.5. Plots of the length against width of the nanorods shown in fig. 2 and fig.4, the fitting curve using equation 4 and aspect ratio calculated based on the fitting data. TP: turning point.

\section{Summary}

In conclusion, we explored the growth mechanism of gold nanorods by adjusting the seed amount, which can tune the aspect ratio of particle effectively. Our results show that during the growth process, gold nanrods, reaching various final morphologies, pass turning point with common size parameters. Further, the kinetics analysis demonstrates that each nanorod in solution grows along the almost same trajectory, which plots the relationship of length and width. The aspect ratio tuning by seed can be ascribed to the growth termination at the different position on the trajectory. This study also indicates of the possibility of quantitative control of gold nanorod aspect ratio.

\section{Acknowledgements}

We thank for the financial support from the National Science Foundation (Grant No. 21565003 and 91233107).

\section{References}

[1] X. Huang, I. H. El-Sayed, W. Qian, M. A. El-Sayed, Cancer Cell Imaging and Photothermal Therapy in the Near-Infrared Region by Using Gold Nanorods, J. Am. Chem. Soc. 128 (2006)2115-2120. 
[2] L. Tong, Y. Zhao, T. B.Huff, M. N.Hansen, A.Wei, J. X. Cheng, Gold Nanorods Mediate Tumor Cell Death by Compromising Membrane Integrity, Adv. Mater. 19(2007)3136-3141.

[3] W. Hou, J. Shao, Q. Qin, Q. Wei, J. Tang, J. Ji, Zwitterionic Phosphorylcholine as a Better Ligand for Gold Nanorods CellUptake and Selective Photothermal Ablation of Cancer Cells, ChemComm. 46(2010)1479-1481.

[4] Durr, N. J. Larson, T. Smith, D. K. Korgel, B. A. Sokolov, K. Ben-Yakar, Two-Photon Luminescence Imaging of Cancer Cells Using Molecularly Targeted Gold Nanorods, Nano Lett. 7(2009)941-945.

[5] X. Wu, T. Ming, X. Wang, P. Wang, J. Wang, J. Chen, High-Photoluminescence-Yield Gold Nanocubes: For Cell Imaging and Photothermal Therapy, ACS nano. 4(2009)113-210.

[6] W. Ahmed, E. S. Kooij, A. V. Silfhout, B. Poelsema, Quantitative Analysis of Gold Nanorod Alignment after Electric Field-Assisted Deposition, Nano. Lett. 9(2009) 3786-3794.

[7] T. Kawano, Y. Niidome, T. Mori, Y.Katayama, A. T. Niidome, PNIPAM Gel-Coated Gold Nanorods for Targeted Delivery Responding to aNear-Infrared Lase, Bioconjugate Chem. 20(2009)209-212.

[8] X. Huang, S. Neretina, M. A. El-Sayed, Gold Nanorods: From Synthesis and Properties toBiological and Biomedical Applications, Adv. Mater. 21 (2009)4880-4910.

[9] T. K. Sau, C. Murphy, Seeded High Yield Synthesis of Short Au Nanorods in Aqueous Solution, Langmuir. 20 (2004) 6414-6420.

[10]S. Si, C. Leduc, M. H. Delville, B. Lounis, Short Gold Nanorod Growth Revisited: The Critical Role of the Bromide Counterion, ChemPhysChem. 13, (2012) 193-202.

[11]K. T. Yong, Y. Sahoo, M. T. Swihart, P. M. Schneeberger, P. N.Prasad, Templated Synthesis of Gold Nanorods (NRs): The Effects of Cosurfactants and Electrolytes on the Shape and Optical Properties, Top Catal. 47(2008) 49-60.

[12]C. K. Tsung, X. Kou, Shi, Q. Zhang, J. Yeung, M. H. Wang, J. Stucky, Selective Shortening of Single-Crystalline Gold Nanorods by Mild Oxidation, J. Am. Chem. Soc. 128 (2006) 5352-5353.

[13]K. C. Ng, W. Cheng, Fine-tuning longitudinal plasmon resonances of nanorods by thermal reshaping in aqueous media, Nanotechnology 23 (2012) 105602.

[14]Y. Wang, S. Long, S. Vdovic, X. F. Wang, Fine Tuning of the Longitudinal Plasmon Resonance of Gold Nanorods by Depleting Gold Precursor, Chem. Mater. 25 (2013) 1372-1376.

[15]Y. Y. Wang, B. X. Li, S. Vdovic, X. F.Wang, A. D. Xia, Kinetic Simulation of Gold Nanorod Growth in Solution Based on Optical Spectra, Chinese J. Chem. Phys. 25 (2012)135-141.

[16]J. Liu, J. N. Duggan, J. Morgan, C. B. Roberts, Seed-mediated growth and manipulation of Au nanorodsvia size-controlled synthesis of Au seeds, J. Nanopart. Res.(2012) 1289.

[17]C. Bullen, P. Zijlstra, E. Bakker, M. Gu, C. Raston, Chemical Kinetics of Gold Nanorod Growth in Aqueous CTAB Solutions, Crystal Growth \&Design. 11 (2011) 3375-3380.

[18]F.Ratto, P.Matteini, F. Rossi, R. Pini, Size and shape control in the overgrowth of gold nanorods, J. Nanopart. Res. 12 (2010) 2029-2036.

[19]Y. J. Xiang, X. C. Wu, D. F. Liu, L. F. Feng, K. Zhang, W. G. Chu, W. Y. Zhou, S. S. Xie, Tuning the Morphology of Gold Nanocrystals by Switching the Growth of $\{110\}$ Facets from Restriction to Preference, J. Phys. Chem. C. 112 (2008) 3203-3208. 
[20]E. Carbo-Argibay, B. Rodriguez-Gonzalez, S. Gomez-Grana, A. Guerrero-Martinez, I. Pastoriza-Santos, J. Perez-Juste, L. M. Liz-Marzan, The Crystalline Structure of Gold Nanorods Revisited: Evidence for Higher-Index Lateral Facets, Angew. Chem. Int. Ed. 49 (2010) 9397-9400. [21]A. Henkel, O. Schubert, A. Plech, C. Sonnichsen, Growth Kinetic of a Rod-Shaped Metal Nanocrystal, J. Phys. Chem. C. 113 (2009) 10390-10394.

[22]M. J. Avrami, Kinetics of Phase Change. II Transformation-Time Relations for Random Distribution of Nuclei, Chem. Phy. 8 (1939) 212-214.

[23]M. J. Avrami, Kinetics of Phase Change. I General Theory, Chem. Phy. 7 (1939) 1103-1112.

[24]C. M. Tollan, J. Echeberria, R.Marcilla, J. A. Pomposo, D. Mecerreyes, One-step Growth of Gold Nanorods Using a b-diketonereducing Agent, J Nanoparticle Res. 11 (2009) 1241-1245.

[25]J. Perez-Juste, L. M. Liz-Marzan, S. Carnie, D. Y. C. Chan, P. Mulvaney, Electric field Directed Growth of Gold Nanorods in Aqueous Surfactant Solutions, Adv. Funct. Mater. 14(2004) 571-579.

[26]L. G. Abdelmoti, F. P. Zamborini, Potential-Controlled Electrochemical Seed-Mediated Growth of Gold Nanorods Directly on Electrode Surfaces, Langmuir. 26 (2010) 13511-13521.

[27]J. I. Stainfeld, J. S.Fransisco, W. L. Hase, Chemical Kinetics and Dynamics, 2nd Edition, Prentice Hall (1998).

[28]H. A. Keul, M. Moller, M. R. Bockstaller, Structural Evolution of Gold Nanorods during Controlled Second Growth, Langmuir. 23 (2007) 10307-10315.

[29]M. Liu, P. Guyot-Sionnest, Mechanism of Silver(I)-Assisted Growth of Gold Nanorods and Bipyramids, J. Phys. Chem. B. 109 (2005) 22192-22200. 